\title{
Inhibition of autophagy in hepatocarcinoma cells promotes chemotherapeutic agent-induced apoptosis during nutrient deprivation
}

\author{
XIAN-LING GUO ${ }^{1,2 *}$, FEI HU ${ }^{2 *}$, HUI WANG ${ }^{2 *}$, JUE-MIN FANG ${ }^{2}$, ZHONG-ZHENG ZHU $^{2}$, \\ LI-XIN WEI ${ }^{3}$ and QING XU ${ }^{1,2}$ \\ ${ }^{1}$ Department of Medical Oncology, Dermatology Hospital, Tongji University, Shanghai 200072; \\ ${ }^{2}$ Department of Medical Oncology, 10th People's Hospital, Tongji University, Shanghai 200072; \\ ${ }^{3}$ Tumor Immunology and Gene Therapy Center, Eastern Hepatobiliary Surgery Hospital, \\ The Second Military Medical University, Shanghai 200438, P.R. China
}

Received April 21, 2017; Accepted October 27, 2017

DOI: $10.3892 /$ or.2017.6115

\begin{abstract}
Autophagy is a lysosome-dependent process involved in protein and organelle degradation. It has been suggested that autophagy is activated in nutrient-deficient condition and plays an important role in protecting cells from nutrient shortage. However, the effect of autophagy on chemotherapy during nutrient deficiency has been rarely researched. In the present study, we discovered that hepatocarcinoma cells exhibit chemoinsensitivity accompanied by the activation of autophagy when cultured in nutrient-deprived medium. Inhibition of autophagy by 3-methyladenine or siRNA-targeted Beclin 1 increased the nutrient deprivation-induced apoptosis and chemosensitivity in hepatocarcinoma cells. Furthermore, decreased mitochondrial mass was detected when cells
\end{abstract}

Correspondence to: $\mathrm{Dr}$ Qing Xu, Department of Medical Oncology, Dermatology Hospital, Tongji University, 1278 Baode Road, Shanghai 200072, P.R. China

E-mail: xuqingmd@aliyun.com

Dr Li-Xin Wei, Tumor Immunology and Gene Therapy Center, Eastern Hepatobiliary Surgery Hospital, The Second Military Medical University, 225 Changhai Road, Shanghai 200438, P.R. China

E-mail: lixinwei@smmu.edu.cn

*Contributed equally

Abbreviations: HCC, hepatocarcinoma; 5-FU, 5-fluorouracil; LC3, microtubule-associated protein 1 light chain 3; DAPI, 4',6'-diamidino2-phenylindole dihydrochloride; 3-MA, 3-methyladenine; TACE, transarterial chemoembolization; GFP-LC3, green fluorescent protein-tagged LC3; PI, propidium iodide; FITC, fluorescein isothiocyannate; GFP, green fluorescent protein; siRNA, small interfering RNA; TEM, transmission electron microscopy

Key words: autophagy, hepatocarcinoma, chemotherapy, apoptosis, nutrient deprivation underwent autophagy. The present study suggests that induction of autophagy confers a survival advantage for hepatocarcinoma cells during nutrient deprivation, not only rescuing cells from nutrient deficiency-induced cell apoptosis, but also protecting cells from chemotherapy-induced cell death. Combined usage of the inhibition of autophagy and conventional chemotherapeutic agents could be an effective therapy for hepatocarcinoma during nutrient deprivation.

\section{Introduction}

Hepatocarcinoma (HCC) is one of the most common malignant tumors in China (1). Surgical resection and liver transplantation are potential curative therapies (2). Unfortunately, most HCCs are too advanced at the time of diagnosis to benefit from these surgical approaches. Currently, chemotherapy is ineffective for HCC due to the inherent chemoresistance. However, the exact mechanism of chemotherapy resistance in HCC is largely unknown. Recently, accumulated evidence suggests that autophagy can promote cancer resistance to chemotherapeutic agents (3-6). Autophagy was first identified as a cellular defense response to nutrient deprivation and is essential to maintaining cell survival during nutrient starvation $(7,8)$. It has been reported that due to the insufficient blood supply following poor vasculature, tumors are able to obtain a limited supply of oxygen and nutrients during their progression (9). Moreover, certain treatments for HCC, such as transarterial chemoembolization (TACE) (10) also result in a nutrient-deficient microenvironment. Thus, it is crucial to explore whether nutrient deprivation-induced autopahgy may contribute to the chemoresistance of HCC.

Autophagy is an evolutionarily conserved physiological process, and can be activated under various stimuli such as starvation and hypoxia (11). The autophagic pathway begins with a lipid bilayer structure called the isolation membrane. The isolation membrane sequesters cytoplasmic materials, such as organelles, to form autophagosomes. Subsequently, the autophagosome fuses with the lysosome and matures into autolysosomes. Sequestrated materials are digested to amino 
acids in the autolysosomes by lysosomal enzymes (12-14). Several pharmacologic autophagy inhibitors have been used to evaluate the physiologic relevance of autophagy in culture cells. For example, 3-methyladenine (3-MA) blocks autophagosome formation to inhibit autophagy (15).

The role of autophagy in cancer is still controversial. For example, prolonged autophagy has been suggested to cause autophagic cell death, which is termed as type II programmed cell death $(16,17)$. In contrast, autophagy allows cancer cells to survive in response to adverse conditions such as nutrient deprivation by providing amino acid and other intermediates. Inhibition of autophagy increases nutrient starvation-induced cell death (18). Although, autophagy is essential for helping cells against a shortage of nutrients, the role of autophagy in chemotherapy during nutrient deprivation has been rarely explored.

In the present study, we report that HCC cells exhibited chemoresistance accompanied by activation of autophagy during nutrient deprivation. Inhibition of autophagy increased nutrient deprivation-induced apoptosis. Decreased mitochondrial mass was detected when cells underwent autophagy. Furthermore, inhibition of autophagy enhanced the chemosensitivity of HCC cells to chemotherapeutic agents.

\section{Materials and methods}

Cell culture. Human hepatocarcinoma cell lines SMMC-7721, Hep3B and HepG2 [obtained from the Tumor Immunology and Gene Therapy Center of the Eastern Hepatobiliary Surgery Hospital (Shanghai, China)] were maintained in Dulbecco's modified Eagle's medium (high glucose) (Gibco, Invitrogen, Carlsbad, CA, USA) and supplemented with $10 \%$ fetal bovine serum, $100 \mathrm{U} / \mathrm{ml}$ penicillin and $100 \mathrm{mg} / \mathrm{ml}$ streptomycin in a humidified incubator under $5 \% \mathrm{CO}_{2}$ at $37^{\circ} \mathrm{C}$. Nutrient deprivation was carried out in Earle's balanced salt solution (EBSS) medium (Sigma-Aldrich, St. Louis, MO, USA).

Regents. Cisplatin and 5-fluorouracil (5-FU) were purchased from Qilu Pharmaceutical Co., Ltd. (Jinan, Shandong, China). 3-MA was obtained from Sigma-Aldrich (Shanghai, China) and dissolved in sterile double distilled water.

Cell viability assay. The measurement of viable cell mass was performed with a Cell Counting Kit (Cell Counting Kit-8, Dojin Laboratories, Kumamoto, Japan) to count living cells using WST-8. Cells were seeded $\left(0.6 \times 10^{4}\right.$ cells/well) on a 96-well plate and $24 \mathrm{~h}$ later, the cells were treated with nutrientcontaining and nutrient-deprived medium with or without the indicated chemotherapeutic agents; in some experiment, 3-MA $(10 \mathrm{mM})$ was added. For quantitative analysis of cell viability, $10 \mu \mathrm{l}$ of cell counting kit solution was added to each well. After incubation at $37^{\circ} \mathrm{C}$ for $2 \mathrm{~h}$ in a humidified $\mathrm{CO}_{2}$ incubator, absorbance at $450 \mathrm{~nm}$ was monitored with a microplate reader (Synergy HT; BioTek Instruments, Inc., Winooski, VT, USA). The values obtained were normalized to those of the control cells incubated with vehicle only.

4',6-Diamidino-2-phenylindole (DAPI) staining. Cells were seeded into a 96 -well plate and $24 \mathrm{~h}$ later cells were subjected with nutrient-containing and nutrient-deprived medium with or without the indicated chemotherapeutic agents; in some experiment, 3-MA was added. For DAPI staining, the cells were fixed with $4 \%$ paraformaldehyde for $10 \mathrm{~min}$ at room temperature. After being washed with 1X PBS for 2 times, the cells were then treated with $0.1 \%$ Triton X-100 for 5 min prior to staining with $1 \mu \mathrm{g} / \mathrm{ml}$ DAPI. Cells were visualized by fluorescence microscopy (Olympus IX71; Olympus, Tokyo, Japan). Cells in which the nuclei contained clearly condensed chromatin or cells exhibiting fragmented nuclei were scored as apoptotic. Apoptotic data are reported as percentage of apoptosis, obtained by determining the numbers of apoptotic cells vs. the total number of cells. For each sample, a minimum of 5 counts involving a minimum of 100-200 cells/count were scored.

Annexin V/PI staining. SMMC-7721, Hep3B and HepG2 cells were allowed to reach $70-80 \%$ confluency, and then were subjected to nutrient-containing and nutrient-deprived medium with or without the indicated chemotherapeutic agents; in some experiment, 3-MA was added. Cells $\left(1 \times 10^{6}\right)$ were collected by trypsinization at the times indicated. All cells were washed with ice-cold phosphate-buffered saline (PBS) twice and resuspended in $300 \mu \mathrm{l} 1 \mathrm{X}$ binding buffer containing $5 \mu \mathrm{l}$ Annexin $\mathrm{V}$ and $5 \mu \mathrm{l} \mathrm{PI}$ for $30 \mathrm{~min}$ at room temperature in the dark (Annexin V-FITC apoptosis detection kit; Nanjing Keygen Biotech, China). Cell survival was measured by flow cytometric analysis using a FACSAria flow cytometer (Becton-Dickinson, Franklin Lakes, NJ, USA). Annexin V and PI single-positive, and double-positive populations were collectively counted as 'dead', whereas double-negative cells were considered viable.

Transient transfection. SMMC-7721, Hep3B and HepG2 cells were seeded $\left(1 \times 10^{4}\right.$ cells/well) into 96 -well plates overnight, and then GFP-LC3-expressing plasmids were transiently transfected into the cells using FuGENE HD transfection reagent (Roche, Mannheim, Germany) according to the manufacturer's instructions. Cultured for $24 \mathrm{~h}$ to ensure the expression of GFP-LC3, the cells were subjected to EBSS medium in the absence or presence of 3-MA. At the end of the treatment, autophagy was detected by counting the percentage of cells with GFP-LC3-positive dots under a fluorescence microscope (Olympus IX71). A minimum of 200 cells/sample were counted in triplicate for each experiment.

Transmission electron microscopy. Cells were fixed with $2.5 \%$ glutaraldehyde in phosphate buffer, and stored at $4^{\circ} \mathrm{C}$ until embedding. Cells were post-fixed with $1 \%$ osmium tetroxide followed by an increasing gradient dehydration step using ethanol and acetone. Cells were then embedded in araldite, and ultrathin section were obtained (50-60 nm), placed on uncoated copper grids, and stained with $3 \%$ lead citrate-uranyl acetate. Images were examined with a CM-120 electron microscope (Philips, Andover, MA, USA).

siRNA. The Stealth ${ }^{\mathrm{TM}}$ RNAi negative control duplex (cat. 12935200) and Stealth ${ }^{\mathrm{TM}}$ RNAi siRNA duplex oligoribonucleotides targeting human Beclin 1 (cat. 1299003) were obtained from Invitrogen. The siRNA was transfected into SMMC-7721 cells using siRNA transfection reagent (cat. sc-29528; Santa Cruz 
Biotechnology, Santa Cruz, CA, USA) according to the manufacturer's protocol.

Western blot analysis. At the end of the designated treatments, cells were lysed in RIPA lysis buffer (Beyotime Biotechnology, Shanghai, China) with $1 \mathrm{mM}$ phenylmethanesulfonyl fluoride (PMSF). Equal amounts of protein were separated by sodium dodecyl sulfate-polyacrylamide gel electrophoresis (SDS-PAGE) and transferred onto an NC membrane. After blocking with $5 \%$ non-fat milk, the membrane was probed with anti-Beclin 1 (Novus Biologicals, Inc., Cat. NB500-249H, Host: Rabbit, dilution 1:1,000), anti-LC3 (Novus Biologicals, Inc. Cat. NB100-2220H, Host: Rabbit, dilution 1:1,000) and anti-PARP (Cell Signaling Technology, Beverly, MA, USA, Cat. 9532, Isotype: Rabbit, dilution 1:1,000), developed with the BeyoECL Plus substrate system (Beyotime, China, Cat. P0018). Blots were stripped and re-probed with $\beta$-actin antibody (Santa Cruz, Cat. sc-47778, Host: mouse, dilution $1: 2,000)$ to confirm equal protein loading.

Semi-quantitative real-time PCR. RNA was extracted from cells using TRIzol (Invitrogen, Carlsbad, CA, USA). cDNA was synthesized using MMLV reverse transcriptase (Promega, Madison, WI, USA), and $2 \mu \mathrm{g}$ total RNA and oligo(dT) primers. Two-microliter aliquots of cDNA were used for PCR amplification. Real-time RT-PCR was performed in triplicate using the SYBR PrimeScript RT-PCR kit (Takara, Dalian, China), and primers used were as follows: sense, 5'-CCATCTTT GCAGGCACACTCATC-3', and antisense 5'-ATCCACCTCA ACTGCCTGCTATG-3' for Nd2 (19); sense, 5'-ACAGCTCGT GTAATCTACCA-3' and antisense, 5'-GACCGTCCATTCTT TGC-3' for Nme1. PCR used 40 cycles of $5 \mathrm{sec}$ at $95^{\circ} \mathrm{C}, 20 \mathrm{sec}$ at $60^{\circ} \mathrm{C}$ for $\mathrm{Nd} 2$ and $\mathrm{Nme} 1$. The expression of $\mathrm{Nmel}$ served as internal control. PCR products were separated by $2 \%$ agarose gel electrophoresis, and bands were visualized under ultraviolet (UV) radiation after staining with ethidium bromide. Gels were photographed and bands were analyzed by computerized densitometry.

Mitochondrialmembrane potentialdetection. The Mitochondrial Membrane Potential Assay kit with JC-1 was obtained from Beyotime Biotechnology and the procedure was carried out according to the manufacturer's instructions, respectively.

Statistical analysis. All of the experiments were repeated at least 3 times. The data are expressed as means \pm SD. Statistical analysis was performed using the Student's t-test (two-tailed). The criterion for statistical significance was taken as $\mathrm{P}<0.05$.

\section{Results}

Chemotherapeutic agent-induced cell death is reduced in HCC cells during nutrient deprivation. To investigate the functional significance of nutrient shortage in chemotherapy, we incubated 3 HCC cell lines (HepG2, Hep3B and SMMC7721) in EBSS medium (nutrient-deprived medium) and assessed the chemosensitivity of these cells. HCC cells were treated with 5-FU $(100 \mu \mathrm{g} / \mathrm{ml})$ or cisplatin $(8 \mu \mathrm{g} / \mathrm{ml})$ during nutrient deprivation for $12 \mathrm{~h}$. As shown in Fig. 1A, WTS-8 assay results revealed that $\mathrm{HCC}$ cells incubated in nutrient-deprived
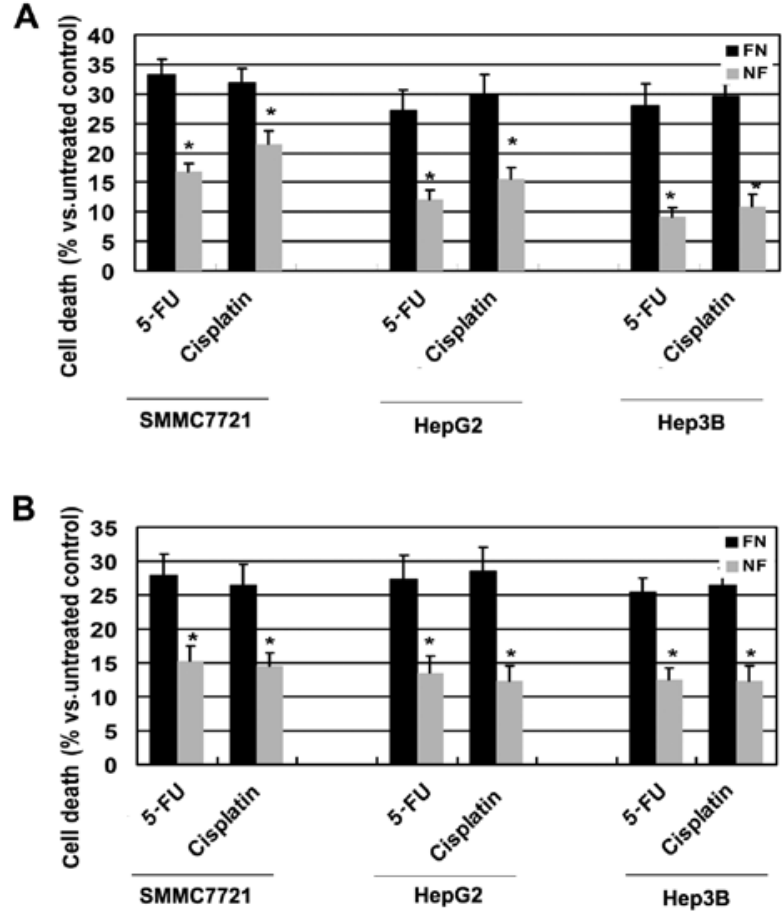

Figure 1. Chemotherapeutic agent-induced cell death is attenuated during nutrient deprivation in hepatocarcinoma cells. Three hepatocarcinoma cell lines (HepG2, Hep3B and SMMC-7721) were cultured in nutrient-free (EBSS medium) or nutrient-containing medium and treated with 5 -FU $(100 \mu \mathrm{g} / \mathrm{ml})$ or cisplatin $(8 \mu \mathrm{g} / \mathrm{ml})$ for $12 \mathrm{~h}$. (A) Cell viability was determined by WST- 8 assay. The viability of the untreated cells was regarded as $100 \%$. Points mean of 3 independent experiments. (B) Cell death was quantified using flow cytometry with Annexin V and propidium iodide double staining as described in Materials and methods. Results (mean \pm SE) represent the average of 3 experiments. FN, full nutrient; NF, nutrient-free. ${ }^{*} \mathrm{P}<0.05$ compared to cells at nutrient-containing medium.

medium exhibited significantly reduced susceptibility to the chemotherapeutic agents, when compared to their counterpart cells which were cultured in nutrient-containing medium. These results were further confirmed by flow cytometric analysis with Annexin V/PI staining. Nutrient-deficient HCC cells were more insensitive to chemotherapeutic agents (Fig. 1B). Thus, these data suggest that HCC cells acquire a protective effect against chemotherapy during nutrient deprivation.

Autophagy is activated in HCC cells during nutrient deprivation. Nutrient deprivation is a strong inducer of autophagy. Thus, we then investigated whether autophagy was activated in HCC cells during nutrient deprivation. LC3, one of the mammalian homologues of yeast ATG8, is activated and relocalizes to intracellular vesicles during the formation of autophagosomes (20). The LC3 pro-form is cleaved into soluble LC3-I, then LC3-I is modified to be membrane-bound, which is known as LC3-II, and recruited onto autophagosomes. LC3-I to LC3-II protein processing is considered a hallmark of autophagy. As shown in Fig. 2A, levels of endogenous LC3-II were markedly increased in the SMMC-7721 cells incubated in nutrient-deprived medium and were attenuated by 3-MA treatment.

To confirm the involvement of autophagy using additional independent assays, 3 HCC cell lines, transfected with the GFP-LC3 plasmid, were cultured for $12 \mathrm{~h}$ in nutrient-deprived medium with or without autophagy inhibitor 3-MA. The 
A

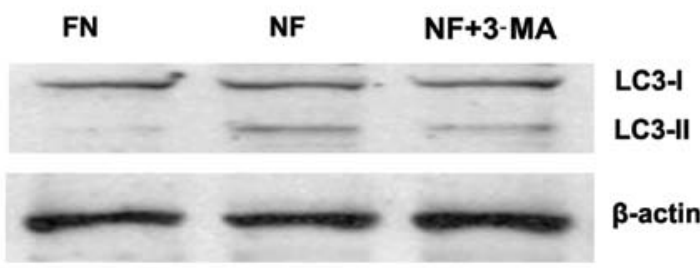

B
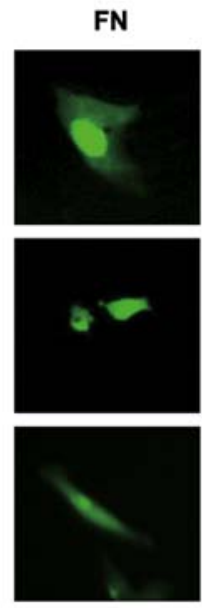

D

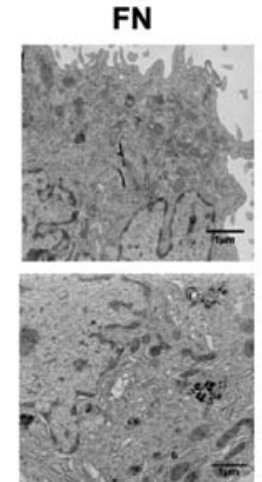

NF
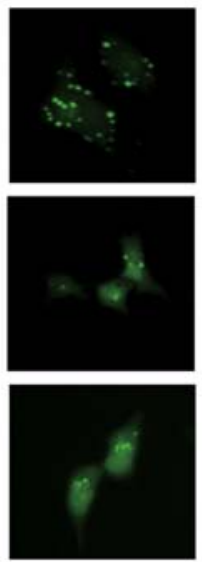
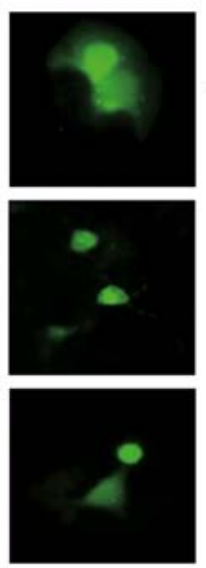

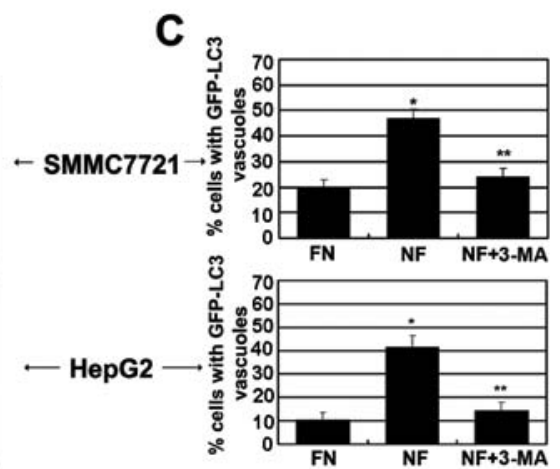

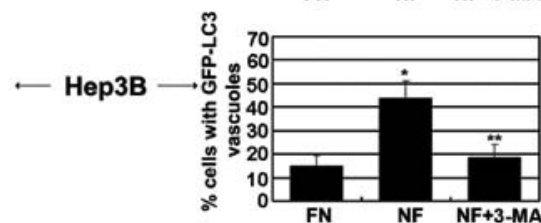

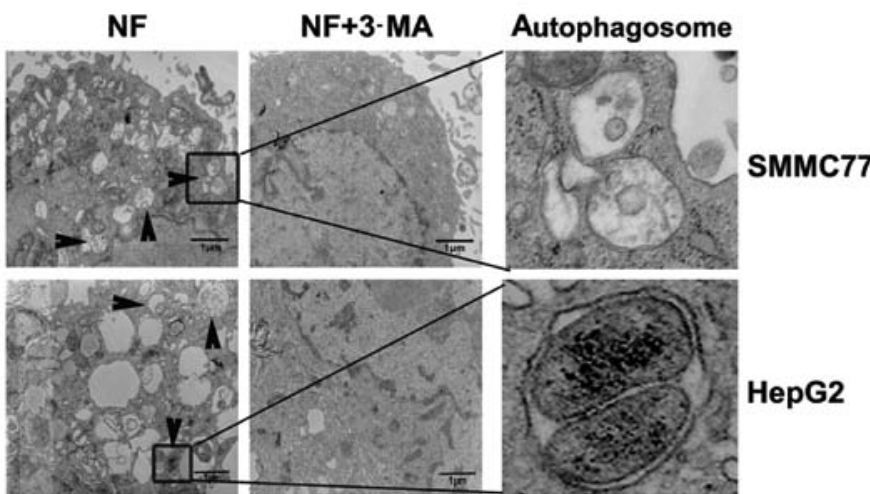

Figure 2. Nutrient deprivation activates autophagy in hepatocarcinoma cells. (A) SMMC-7721 cells were cultured in nutrient-free or nutrient-containing medium with or without autophagy inhibitor 3-MA (10 mM), and whole cell lysates were subjected to western blotting for LC3. (B and C) Hepatocarcinoma cells were transfected with the GFP-LC3 plasmid, and then were cultured in nutrient-free or nutrient-containing medium with or without autophagy inhibitor 3-MA (10 mM). Autofluorescence of GFP-LC3 was evaluated by fluorescence microscopy. (B) Representative images are shown. The percentages of cells with punctuate GFP-LC3 fluorescence were evaluated relative to all GFP-positive cells. Data (mean \pm SD) represent the mean of at least 3 independent experiments. (C) Cells presenting $>2$ autophagosomes/cell were scored as having an autophagic reaction; ${ }^{*} \mathrm{P}<0.05$ compared to cells in nutrient-containing medium, ${ }^{* *} \mathrm{P}<0.05$ compared to cells in nutrient-free medium. (D) Representative electron microscopic images are shown. An arrow indicates autophagosome. The high magnification image (the column labeled autophagosome) shows that the autophagic vacuoles contained electron dense material and degraded subcellular organelles. FN, full nutrient; NF, nutrient-free.

distribution of GFP-LC3 was determined by fluorescence microscopy. As shown in Fig. 2B and C, cells cultured in nutrient-deprived medium exhibited a significantly higher percentage of punctuate GFP (green dots), while nutrientcontaining cells showed primarily diffusion. Furthermore, 3-MA, which inhibits autophagosome formation, significantly reduced the visible green dots in the nutrient-deprived cells and redistributed GFP-LC3 to the cytoplasm. The morphological hallmark of autophagy is the formation of a double-layered membrane structure, termed autophagosome. To date, transmission electron microscopy is the gold standard to monitor the formation of autophagosomes. As shown in Fig. 2D, increased autophagosomes were observed in the nutrient- deprived HCC cells, and 3-MA treatment largely reduced the amount of autophagosomes. In conclusion, these findings suggest that autophagy was activated in these HCC cells under nutrient-deprived condition.

Inhibition of autophagy by 3-MA increases chemosensitivity of HCC cells during nutrient deprivation. Autophagy can be triggered by nutrient deprivation and plays a role in protecting cells from nutrient shortage. However, the role of autophagy in chemotherapy during nutrient starvation has rarely been explored. Therefore, we first tested whether autophagy contributes to cell escape from nutrient shortage-induced cell death. $\mathrm{HCC}$ cells were cultured for $12 \mathrm{~h}$ in nutrient-deprived medium 
A

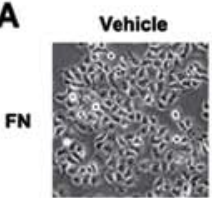

3-MA
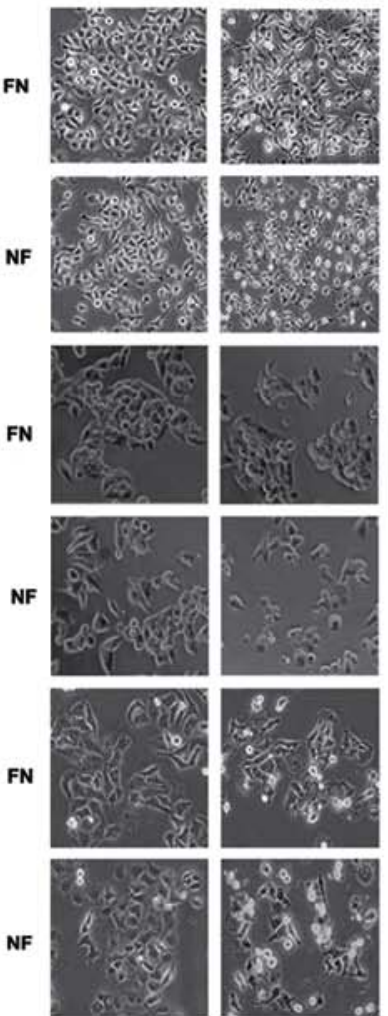
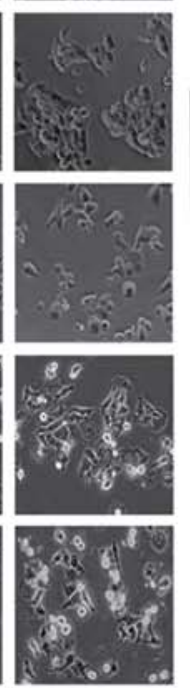

B

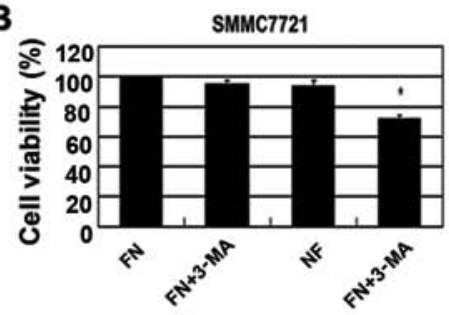

Hep62

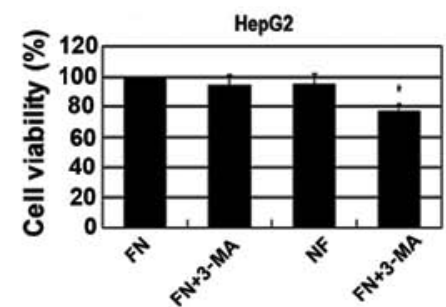

Hep3B

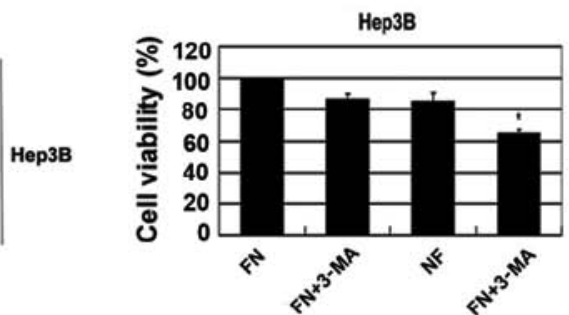

C
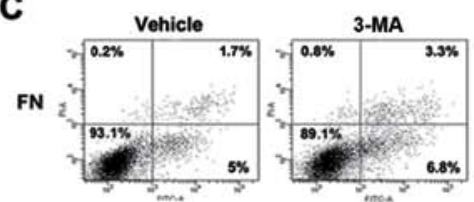

NF
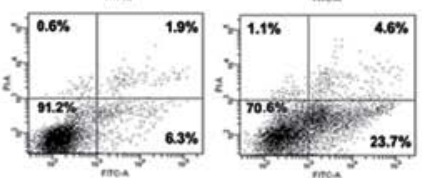

SMMC7721
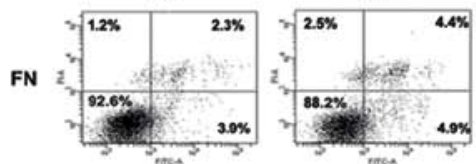

NF
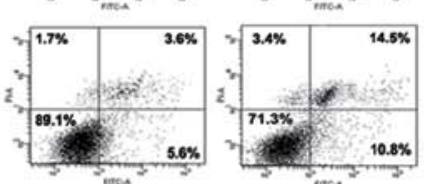

HepG2
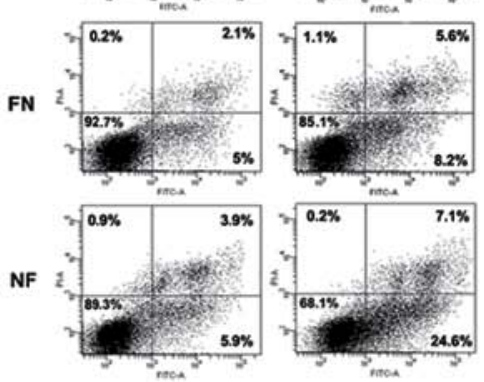

D

FN

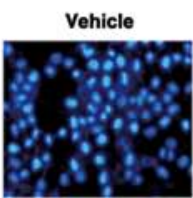

NF

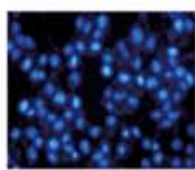

FN

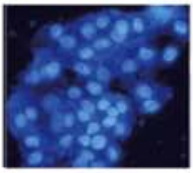

NF

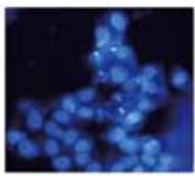

FN

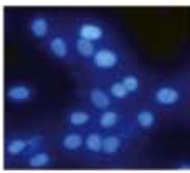

NF

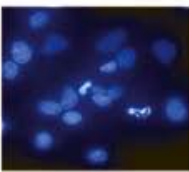

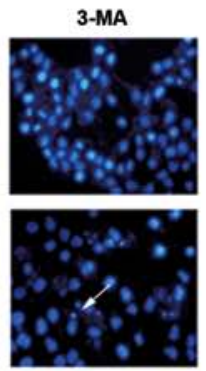
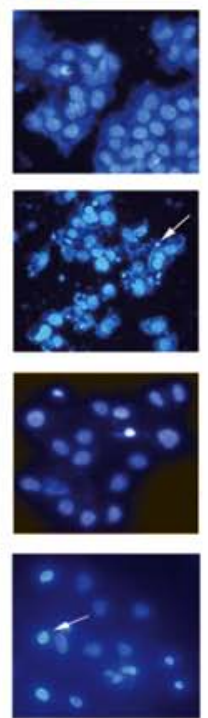
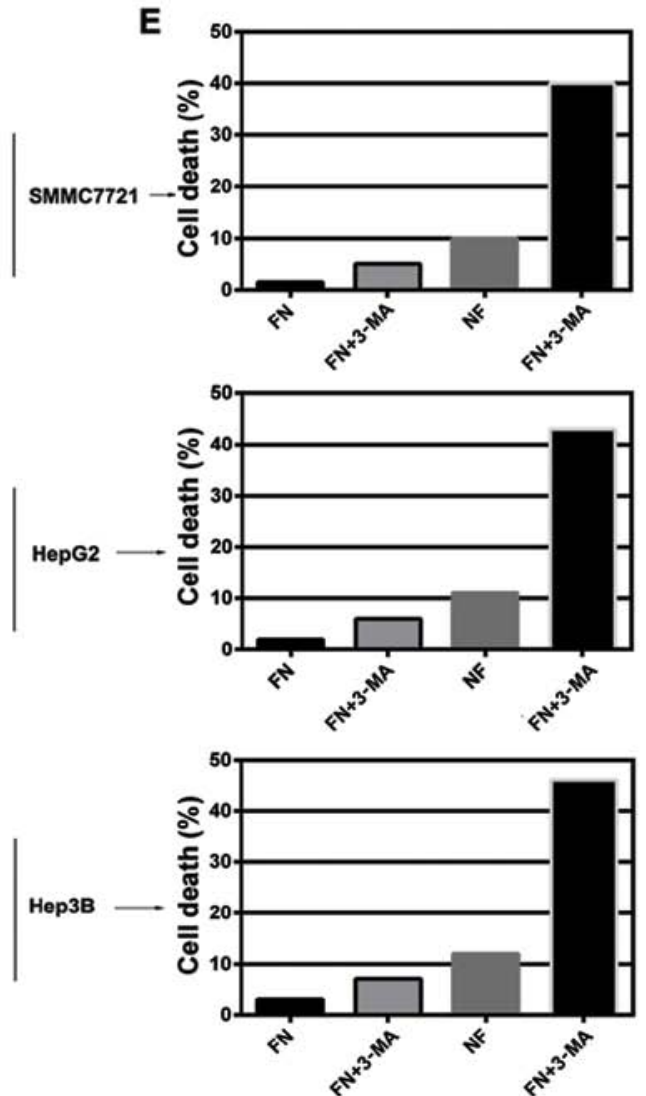

Figure 3. Inhibition of autophagy by 3-MA enhances nutrient deprivation-induced apoptosis in hepatocarcinoma cells. Hepatocarcinoma cells were cultured in nutrient-free or nutrient-containing medium with or without autophagy inhibitor 3-MA. (A) The morphology of cells was observed, and images were captured under a microscope. Representative morphologic images are shown. (B) Cell viability was determined by WST-8 assay. The viability of the untreated cells was regarded as $100 \%$. Data (mean $\pm \mathrm{SD}$ ) represent the means of at least 3 independent experiments; ${ }^{*} \mathrm{P}<0.05$ compared to cells in nutrient-free media. FN, full nutrient; NF, nutrient-free. (C) Cell death was quantified using flow cytometry with Annexin V and propidium iodide double staining as described in Materials and methods. Results (mean \pm SE) represent the average of 3 experiments. (D) DAPI staining was used to identify morphological features of cell apoptosis such as nuclear condensation and fragmentation. (E) Quantitative analysis of cell apoptosis in D. FN, full nutrient, NF, nutrient-free. 
A

SMMC7721
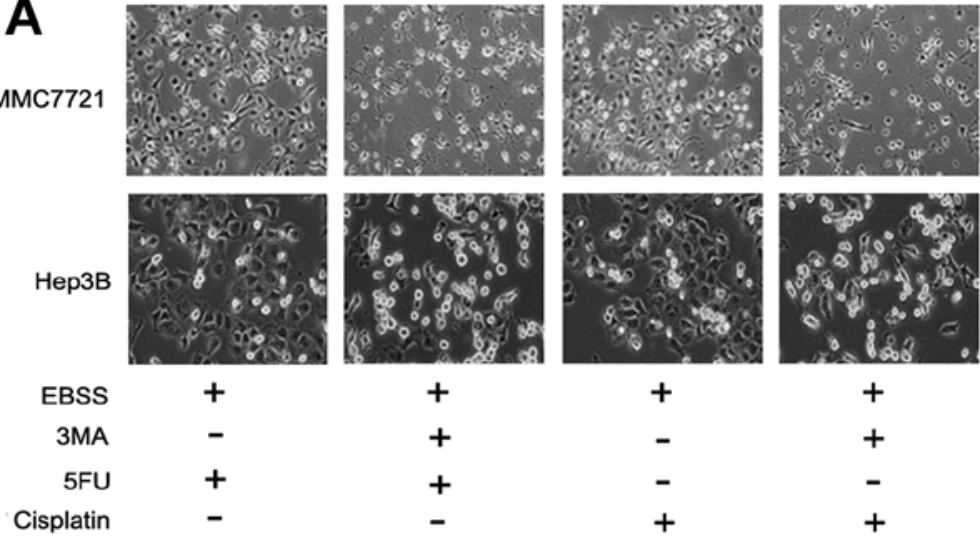

$+$

$+$

$+$
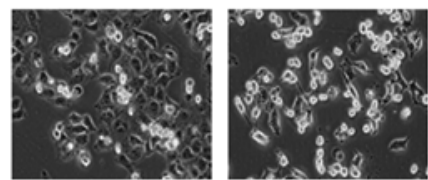

$+$

$-$

-

$-$

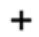

$+$

$+$

$+$

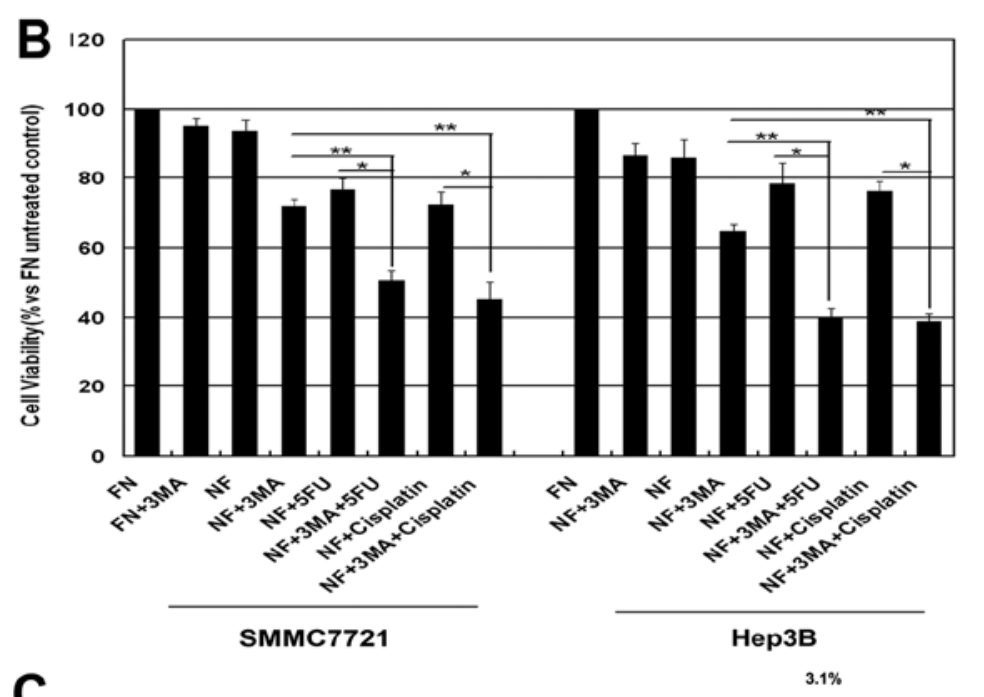

C
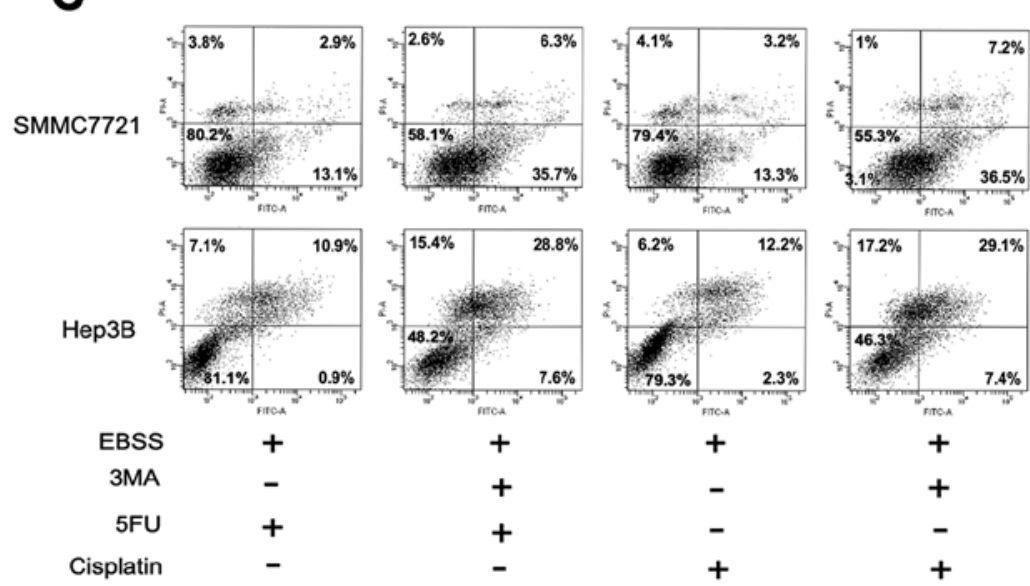

+
+

$+$

$-$

Figure 4. Inhibition of autophagy by 3-MA amplifies chemotherapeutic agent-induced cell death during nutrient deprivation in hepatocarcinoma cells. Hep3B and SMMC-7721 cells were treated with 3-MA in the presence of 5-FU $(100 \mu \mathrm{g} / \mathrm{ml})$ and cisplatin $(8 \mu \mathrm{g} / \mathrm{ml})$ for $12 \mathrm{~h}$ during nutrient deprivation. (A) The morphology of cells was observed, and images were captured under a microscope. Representative morphologic images are shown. (B) Cell viability was determined by WST-8 assay. The viability of the untreated cells was regarded as $100 \%$. Data (mean $\pm \mathrm{SD}$ ) represent the means of at least 3 independent experiments. ${ }^{*} \mathrm{P}<0.05$ compared to cells without 3-MA, ${ }^{* *} \mathrm{P}<0.05$ compared to cells without chemotherapeutic agent. (C) Cell death was quantified using flow cytometry with Annexin $\mathrm{V}$ and propidium iodide double staining as described in Materials and methods. Results (mean $\pm \mathrm{SE}$ ) represent the average of 3 experiments. FN, full nutrient; NF, nutrient-free.

and the autophagy inhibitor (3-MA) was applied to examine the viability of the cells. Cell morphology was detected by inverted phase contrast microscope. Typical apoptotic changes were observed in 3-MA-treated cells during nutrient deprivation, including marked rounding, shrinkage and detachment from the culture dish (Fig. 3A). These results were confirmed by WTS-8 assay. 3-MA treatment resulted in significantly increased cell death (Fig. 3B). Similar results were further obtained by Annexin V/PI staining analysis; 3-MA treatment in nutrient-deprived medium induced significantly increased the apoptosis of cells (Fig. 3C). DAPI staining also demonstrated that 3-MA treatment markedly induced chromatin condensa- 
A

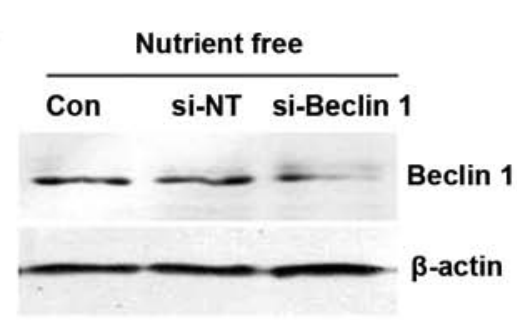

B

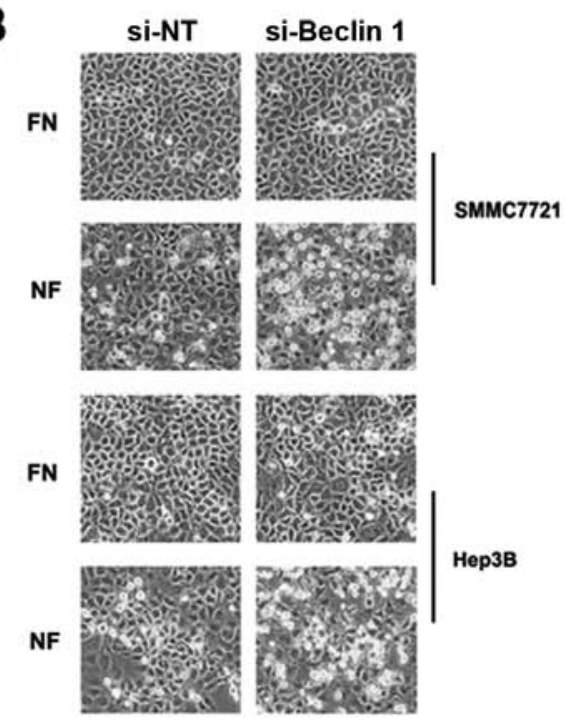

C

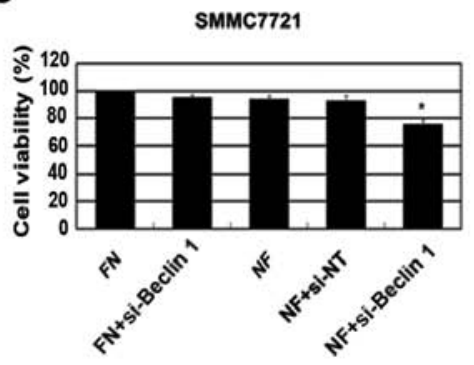

Hep3B

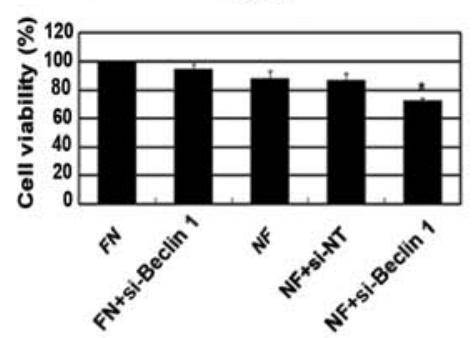

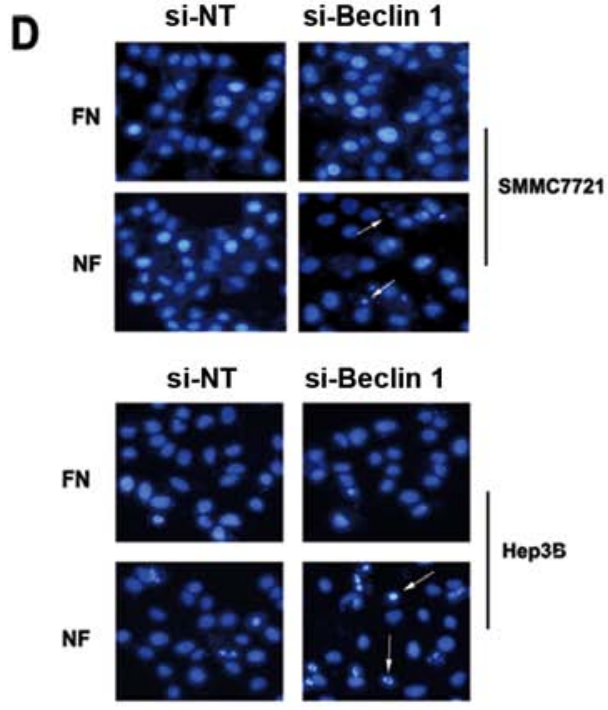

E
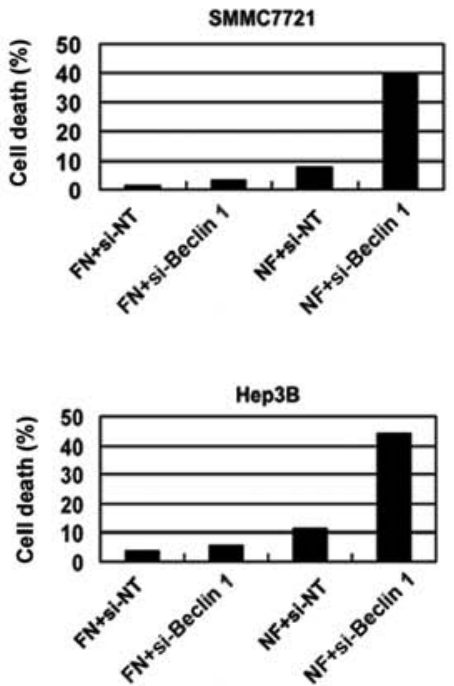

Figure 5. Inhibition of autophagy by si-Beclin 1 increases nutrient deprivation-induced apoptosis in hepatocarcinoma cells. (A) SMMC-7721 cells were transfected with siRNA negative control or siRNA for Beclin 1 for $36 \mathrm{~h}$ followed by incubated in nutrient-free medium for $12 \mathrm{~h}$, and whole cell lysates were subjected to western blotting for Beclin 1. siNT (si-non-target), RNAi negative control. (B) The morphology of cells was observed, and images were captured under a microscope. Representative morphologic images are shown. (C) Cell viability was determined by WST- 8 assay. The viability of the untreated cells was regarded as $100 \%$. Data (mean $\pm \mathrm{SD}$ ) represent the means of at least 3 independent experiments; ${ }^{*} \mathrm{P}<0.05$ compared to cells at NF+si-NT. FN: full nutrient, NF: nutrient-free. (D) DAPI staining was used to identify morphological features of cell apoptosis such as nuclear condensation and fragmentation. (E) Quantitative analysis of cell apoptosis in D. FN, full nutrient; NF, nutrient-free.

tion in HCC cells during nutrient deprivation (Fig. 3D and E). The concentration of these inhibitors did not affect the cell viability in nutrient-containing medium. These data suggested that inhibition of autophagy by 3-MA resulted in increased apoptosis in HCC cells during nutrient deprivation.

To determine whether inhibition of autophagy by 3-MA enhanced the chemotherapeutic sensitivity of the HCC cells, Hep3B and SMMC-7721 cells were treated with 3-MA in the presence of the chemotherapeutic agents, 5-FU or cisplatin during nutrient deprivation. As shown in Fig. 4A, a significantly increased amount of cell death was observed following the combination treatment of 3-MA and 5-FU or cisplatin by observation of cell morphology. WTS-8 assay also showed that the combination treatment (3-MA and 5-FU or cisplatin) exhibited a much greater extent of cell death (Fig. 4B).
Similar effects were further confirmed by Annexin V/PI staining analysis; co-treatment of 3-MA and 5-FU or cisplatin induced significantly more robust cell death under the same conditions (Fig. 4C). DAPI staining also demonstrated that 3-MA treatment markedly induced chromatin condensation in HCC cells following treatment with the chemotherapeutic agents during nutrient deprivation (data not shown). These results were further determined by immunoblotting, where the combination treatment caused an increased level of cleaved PARP in the SMMC-7721 cells (data not shown). JC-1 staining also confirmed that the combination treatment (3-MA and 5-FU or cisplatin) caused a significant loss of mitochondrial membrane potential (data not shown). These results suggested that autophagy contributes to chemotherapy insensitivity in HCC cells during nutrient deprivation. 
A
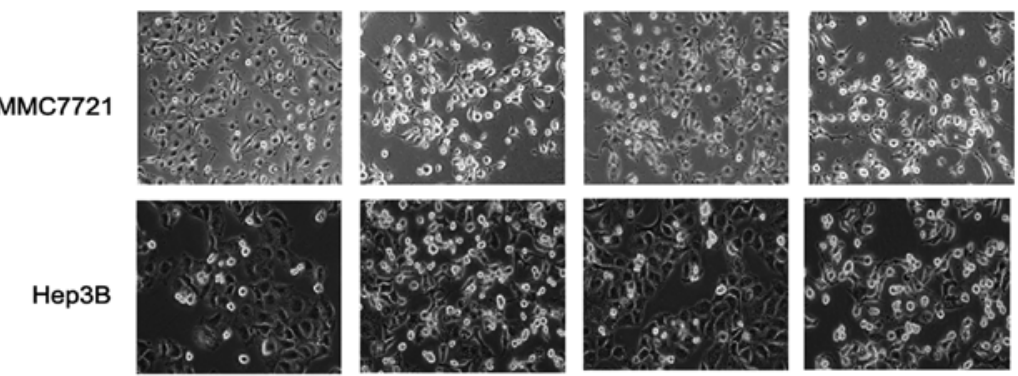

EBSS

si-Beclin 1

$5-\mathrm{FU}$

Cisplatin

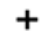

$+$

$-$
$+$

$+$

$+$

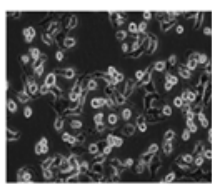

$+$

$+$

$-$

$+$

B

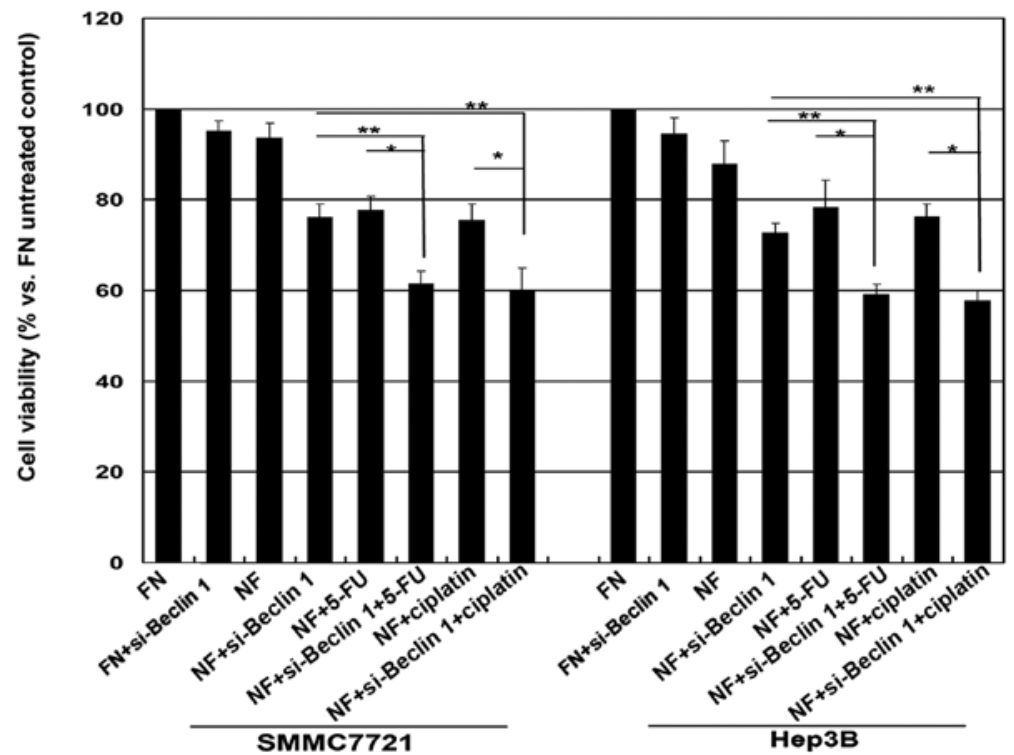

C
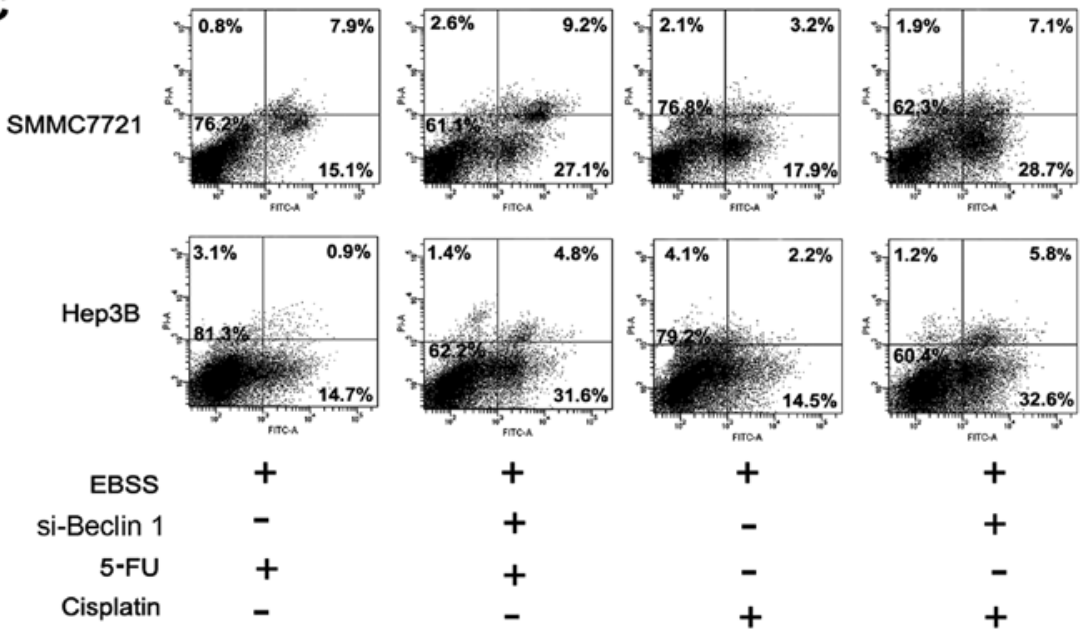

Figure 6. Inhibition of autophagy by si-Beclin 1 enhances chemotherapeutic agent-induced cell death during nutrient deprivation in hepatocarcinoma cells. Hep3B and SMMC-7721 cells were transfected with siRNA for Beclin 1 in the presence of 5-FU (100 $\mu \mathrm{g} / \mathrm{ml})$ and cisplatin $(8 \mu \mathrm{g} / \mathrm{ml})$ for $12 \mathrm{~h}$ during nutrient starvation. (A) The morphology of cells was observed, and images were captured under a microscope. Representative morphologic images are shown. (B) Cell viability was determined by WST- 8 assay. The viability of the untreated cells was regarded as 100\%. Data (mean \pm SD) represent the means of at least 3 independent experiments. ${ }^{*} \mathrm{P}<0.05$ compared to cells without si-Beclin $1,{ }^{* *} \mathrm{P}<0.05$ compared to cells without chemotherapeutic agent. (C) Cell death was quantified using flow cytometry with Annexin $\mathrm{V}$ and propidium iodide double staining as described in Materials and methods. Results (mean $\pm \mathrm{SE}$ ) represent the average of 3 experiments. FN, full nutrient; NF, nutrient-free.

Knockdown of Beclin 1 by specific siRNA enhances chemosensitivity of HCC cells during nutrient deprivation. To confirm the role of the autophagic machinery in nutrient deprivation-induced cell apoptosis, we used siRNA to inhibit the Beclin 1 gene, essential to autophagosome generation $(21,22)$. Silencing of Beclin 1 (Fig. 5A) significantly increased nutrient 
A

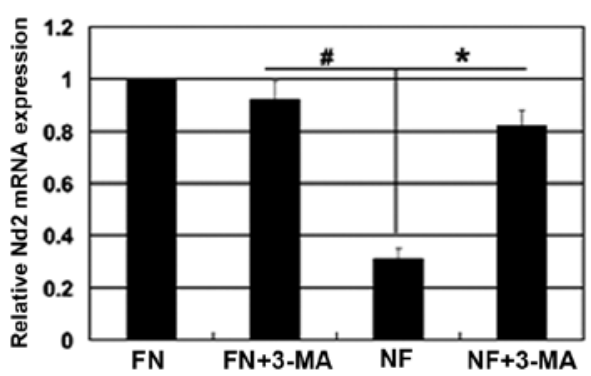

B

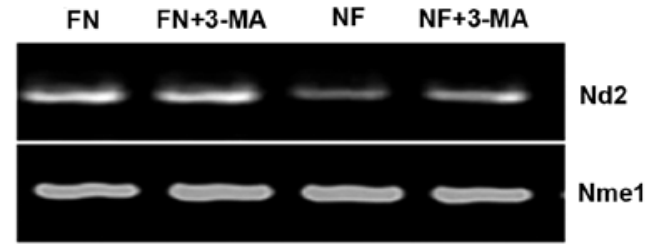

Figure 7. Effect of nutrient deprivation-induced autophagy on mitochondria in HCC cells. (A) Hepatocarcinoma cell line SMMC-7721 was cultured in nutrient-free or nutrient-containing medium with or without autophagy inhibitor 3-MA. Cells were collected, and total RNA was prepared and real-time PCR assays were performed to detect Nd 2 and Nem1 mRNA levels. The Nd 2 mRNA expression levels were normalized to those of Nem1 and are the means \pm SD of at least 3 independent experiments; ${ }^{\#} \mathrm{P}<0.05$ compared to cells in nutrient-containing + 3-MA medium; ${ }^{*} \mathrm{P}<0.05$ compared to cells in nutrient-free + 3-MA medium. (B) PCR products were separated by $2 \%$ agarose gel electrophoresis, and bands were visualized under ultraviolet (UV) radiation after staining with ethidium bromide. A representative example of an experiment that was repeated 3 times is shown.

deprivation-induced cell death (Fig. 5B and C). DAPI staining revealed that silencing of Beclin 1 induced cell apoptosis in the HCC cells (Fig. 5D and E). Together, those data strongly suggest that $\mathrm{HCC}$ cells respond to nutrient shortage by activating autophagy to resist apoptosis.

To further confirm that inhibition of autophagy enhances chemotherapy-induced apoptosis, HCC cells were treated with chemotherapeutic agents for $12 \mathrm{~h}$ after exposure to Beclin 1 siRNA. Knockdown of Beclin 1 significantly augmented chemotherapeutic agent-induced apoptosis (Fig. 6), suggesting that this pathway promotes drug resistance. Together with the findings in Figs. 1 and 4, these results suggest that autophagy contributes to chemotherapy resistance in HCC cells during nutrient deprivation. Thus, nutrient deprivation-induced autophagy in HCC cells is a survival mechanism which protects cells from nutrient shortage and chemotherapy.

Effect of nutrient deprivation-induced autophagy on mitochondria in HCC cells. Autophagy is known as the only pathway for degrading organelles, such as mitochondria (8). Previous studies have shown that decreased mitochondrial mass by autophagy may be accompanied by reduced levels of apoptosis induced by pro-apoptotic agents (23-25). To characterize the mechanism by which autophagy promotes the survival of HCC cells during nutrient deprivation, we tested whether enhanced autophagy during nutrient deprivation can reduce mitochondrial mass in HCC cells. SMMC-7721 cells were cultured for $12 \mathrm{~h}$ in nutrient-deprived medium with or without 3-MA. The expression level of mitochondrial gene $N d 2$ was used to indirectly measure mitochondria mass. Mnel gene was used as the internal control. As shown in Fig. 7A and B, Nd2 mRNA expression was significantly reduced in the SMMC-7721 cells during nutrient deprivation, and significantly restored when 3-MA was used. Moreover, ultrastructural analyses by transmission electron microscopy (TEM) showed that the mitochondrion is enclosed by double membrane-bound vacuolar structures in HepG2 cells (Fig. 2D). Thus, the changes of mitochondria may be a critical step for autophagy inhibitioninduced cell apoptosis.

\section{Discussion}

Autophagy plays an important role in maintaining cellular homeostasis and facilitates cell survival against adverse conditions, such as nutrient deprivation. However, the role of nutrient deprivation-induced autophagy in chemotherapy is poorly understood. In the present study, we showed that autophagy performs a vital role in hepatocarcinoma (HCC) cell survival during nutrient deprivation, not only since it protects cells from nutrient deprivation-induced apoptosis, but also provides cellular protection from exposure to chemotherapeutic agents. In the present study, we found that HCC cells cultured in nutrient-deficient medium showed more resistance to chemotherapeutic agents than their counterparts in nutrient-containing medium. Meanwhile, nutrient-deficient HCC cells exhibited the increased autophagy activity, as is shown by elevated levels of endogenous LC3-II, increased amount of characteristic green dots in the cytoplasm and massive accumulation of autophagosomes; inhibition of autophagy by 3-MA apparently decreased formation of the green dots and the accumulation of LC3II and autophagosomes. In summary, these data indicated that HCC cells exhibited chemoresistance accompanied by autophagy activation under nutrient deprivation.

Recently, several studies have suggested that autophagy plays an important role in maintaining cell survival during nutrient deficiency (26). Consistent with those findings, we observed that autophagy directly contributes to the survival of HCC cells in response to the shortage of nutrients. Nutrient-starved HCC cells treated with an inhibitor of autophagy (3-MA or si-Beclin 1) underwent mass cell death. The results of cell viability assays indicated that the increased cell death by autophagy inhibition was associated by increased apoptosis. Thus, the survival mechanism in HCC during nutrient deprivation is likely due to the reduced apoptotic potential of the cells.

Our previous data showed that HCC exhibited properties of chemoresistance accompanied by autophagy activation under nutrient deprivation. Therefore, we tested the effect of autophagy inhibition on chemosensitivity. We observed that inhibition of autophagy by 3-MA significantly enhanced sensitivity of the HCC cells to cisplatin and 5-FU. Identical data were obtained by inhibiting autophagy with siRNA against Beclin 1. These data implied that autophagy mediates the chemoresistance of HCC cells under nutrient deprivation. These results can be supported by several studies suggesting that autophagy-delayed apoptosis may contribute to drug resistance $(3,27)$. The relationship between autophagy and apoptosis is quite complicated. Under some stress condition, autophagy restrains stress-induced apoptosis to facilitate tumor cell survival (28). Moreover, several studies have demonstrated that autophagy may promote apoptosis (29). Meanwhile, 
in a certain context, autophagy and apoptosis could be simultaneously activated by the same stimulus without any connection (30). Taken together, our current data suggest that induction of autophagy confers two advantages for survival of HCC cells during nutrient deprivation. One is rescuing cells from nutrient deficiency-induced cell apoptosis, and the other is keeping cells from chemotherapy-induced cell death.

What is the functional relevance of nutrient deprivationinduced autophagy activation in HCC? Recent studies have revealed that tumors always suffer the adversity of nutrient deficiency, even after the construction of tumor vessels $(31,32)$. This phenomenon suggests that tumor cells including HCC may physiologically face a shortage of nutrients during development. Moreover, treatment of HCC, such as TACE and TAE (10) also leads to nutrient deficiency. Thus, nutrient shortage-induced autophagy is commonly activated in HCC. Although the role of autophagy in tumors is controversial, the present study clearly showed that autophagy is the primary cell survival mechanism following nutrient deficiency in HCC cells. It has been well demonstrated that failure to induce cell death by anticancer treatment contributes to chemotherapeutic failure and tumor progression. Thus, the present study indicates that in a nutrient deficient microenvironment, activation of autophagy may be an adaptive and a protective mechanism for HCC development, and the combination of the inhibition of autophagy and conventional chemotherapeutic agents could be an effective therapy for HCC. Nutrient deprivation is a type of metabolic stress that causes ROS accumulation. The relationship between ROS and autophagy is quite complicated. ROS, particularly mitochondrial ROS, could induce autophagy activation $(33,34)$. However, removal of ROS by induction of autophagy could promote cell survival (35). In the present study, the role of ROS in autophagy activation and HCC cell survival needs further studies.

Although autophagy was suggested by numerous studies that it can promote cell survival in response to adverse conditions such as nutrient deprivation, the mechanism underlying the effect of autophagy on promoting cell survival remains poorly defined. It has been suggested that clearance of mitochondria and then prevention of the diffusion of pro-apoptotic factors, such as cytochrome $c$, may help cells to escape apoptosis in response to cell death stimuli $(23,24)$. In the present study, we observed that the mitochondria mass was significant decreased under nutrient-deficient condition, and inhibition of autophagy by 3-MA distinctly restored the number of mitochondria. These results suggest that enhanced autophagy may reduce mitochondrial mass. Although it is possible that there exist other factors that contribute to the survival of HCC cells during nutrient deprivation (36), we provide a persuasive data to suggest that reduced mitochondrial mass by activation of autophagy may play a beneficial role in promoting HCC cell survival under nutrient deprivation.

Taken together, the present study suggests that enhanced autophagy protects HCC cells from nutrient deprivation and chemotherapy-induced cell death. This is likely consistent with decreased mitochondrial mass. The physiologic roles of autophagy in tumor progression may closely connect with their microenvironments. These results further expand our understanding of the relevant role of autophagy in cancer formation and progression and may provide new strategies for HCC treatment. It may be reasonable to predict that drugs impairing the nutrient deprivation-induced autophagy pathway may be beneficial for chemotherapy of HCC during TACE. We also would like to extend the present study to other cancers. Further studies on the molecular mechanism by which autophagy promotes chemoresistance are warranted, and may possibly facilitate cancer therapy.

\section{Acknowledgements}

The present study was supported by the National Natural Science Foundation of China (no. 81472623).

\section{References}

1. Tang ZY: Hepatocellular carcinoma - cause, treatment and metastasis. World J Gastroenterol 7: 445-454, 2001.

2. Bartlett A and Heaton N: Hepatocellular carcinoma: Defining the place of surgery in an era of organ shortage. World $\mathbf{J}$ Gastroenterol 14: 4445-4453, 2008.

3. Abedin MJ, Wang D, McDonnell MA, Lehmann U and Kelekar A: Autophagy delays apoptotic death in breast cancer cells following DNA damage. Cell Death Differ 14: 500-510, 2007.

4. Katayama M, Kawaguchi T, Berger MS and Pieper RO: DNA damaging agent-induced autophagy produces a cytoprotective adenosine triphosphate surge in malignant glioma cells. Cell Death Differ 14: 548-558, 2007.

5. Kaushal GP, Kaushal V, Herzog C and Yang C: Autophagy delays apoptosis in renal tubular epithelial cells in cisplatin cytotoxicity. Autophagy 4: 710-712, 2008.

6. Amaravadi RK, Yu D, Lum JJ, Bui T, Christophorou MA, Evan GI, Thomas-Tikhonenko A and Thompson CB: Autophagy inhibition enhances therapy-induced apoptosis in a Myc-induced model of lymphoma. J Clin Invest 117: 326-336, 2007.

7. Maiuri MC, Zalckvar E, Kimchi A and Kroemer G: Self-eating and self-killing: Crosstalk between autophagy and apoptosis. Nat Rev Mol Cell Biol 8: 741-752, 2007.

8. Levine B and Yuan J: Autophagy in cell death: An innocent convict? J Clin Invest 115: 2679-2688, 2005.

9. Ogier-Denis E and Codogno P: Autophagy: A barrier or an adaptive response to cancer. Biochim Biophys Acta 1603: 113-128, 2003.

10. Qian J, Feng GS and Vogl T: Combined interventional therapies of hepatocellular carcinoma. World J Gastroenterol 9: 1885-1891, 2003.

11. Levine B and Klionsky DJ: Development by self-digestion: Molecular mechanisms and biological functions of autophagy. Dev Cell 6: 463-477, 2004.

12. Levine B and Kroemer G: Autophagy in the pathogenesis of disease. Cell 132: 27-42, 2008.

13. Kondo Y, Kanzawa T, Sawaya R and Kondo S: The role of autophagy in cancer development and response to therapy. Nat Rev Cancer 5: 726-734, 2005.

14. Kroemer G and Jäättelä M: Lysosomes and autophagy in cell death control. Nat Rev Cancer 5: 886-897, 2005.

15. Klionsky DJ, Abeliovich H, Agostinis P, Agrawal DK, Aliev G, Askew DS, Baba M, Baehrecke EH, Bahr BA, Ballabio A, et al: Guidelines for the use and interpretation of assays for monitoring autophagy in higher eukaryotes. Autophagy 4: 151-175, 2008.

16. Yu SW, Baek SH, Brennan RT, Bradley CJ, Park SK, Lee YS, Jun EJ, Lookingland KJ, Kim EK, Lee H, et al: Autophagic death of adult hippocampal neural stem cells following insulin withdrawal. Stem Cells 26: 2602-2610, 2008.

17. Gozuacik D and Kimchi A: Autophagy as a cell death and tumor suppressor mechanism. Oncogene 23: 2891-2906, 2004.

18. Sato K, Tsuchihara K, Fujii S, Sugiyama M, Goya T, Atomi Y, Ueno T, Ochiai A and Esumi H: Autophagy is activated in colorectal cancer cells and contributes to the tolerance to nutrient deprivation. Cancer Res 67: 9677-9684, 2007.

19. De Flora S, Scarfì S, Izzotti A, D'Agostini F, Chang CC, Bagnasco M, De Flora A and Trosko JE: Induction by 7,12-dimethylbenz $(a)$ anthracene of molecular and biochemical alterations in transformed human mammary epithelial stem cells, and protection by $N$-acetylcysteine. Int J Oncol 29: 521-529, 2006. 
20. Tanida I, Minematsu-Ikeguchi N, Ueno T and Kominami E: Lysosomal turnover, but not a cellular level, of endogenous LC3 is a marker for autophagy. Autophagy 1: 84-91, 2005.

21. Pattingre S, Tassa A, Qu X, Garuti R, Liang XH, Mizushima N, Packer M, Schneider MD and Levine B: Bcl-2 antiapoptotic proteins inhibit Beclin 1-dependent autophagy. Cell 122: 927-939, 2005.

22. Cao Y and Klionsky DJ: Physiological functions of Atg6/Beclin 1: A unique autophagy-related protein. Cell Res 17: 839-849, 2007.

23. Colell A, Ricci JE, Tait S, Milasta S, Maurer U, Bouchier-Hayes L, Fitzgerald P, Guio-Carrion A, Waterhouse NJ, Li CW, et al: GAPDH and autophagy preserve survival after apoptotic cytochrome $c$ release in the absence of caspase activation. Cell 129 : 983-997, 2007.

24. Ravikumar B,BergerZ, Vacher C,O'Kane CJ and Rubinsztein DC: Rapamycin pre-treatment protects against apoptosis. Hum Mol Genet 15: 1209-1216, 2006.

25. Zhang H, Bosch-Marce M, Shimoda LA, Tan YS, Baek JH, Wesley JB, Gonzalez FJ and Semenza GL: Mitochondrial autophagy is an HIF-1-dependent adaptive metabolic response to hypoxia. J Biol Chem 283: 10892-10903, 2008.

26. Amaravadi RK and Thompson CB: The roles of therapy-induced autophagy and necrosis in cancer treatment.Clin Cancer Res 13: 7271-7279, 2009

27. Bauvy C, Gane P, Arico S, Codogno P and Ogier-Denis E: Autophagy delays sulindac sulfide-induced apoptosis in the human intestinal colon cancer cell line HT-29. Exp Cell Res 268: 139-149, 2001

28. Viola G, Bortolozzi R, Hamel E, Moro S, Brun P, Castagliuolo I, Ferlin MG and Basso G: MG-2477, a new tubulin inhibitor, induces autophagy through inhibition of the Akt/mTOR pathway and delayed apoptosis in A549 cells. Biochem Pharmacol 83: $16-26,2012$.
29. Liao A, Hu R, Zhao Q, Li J, Li Y, Yao K, Zhang R, Wang H, Yang W and Liu Z: Autophagy induced by FTY720 promotes apoptosis in U266 cells. Eur J Pharm Sci 45: 600-605, 2012.

30. Zhang YH, Wu YL, Tashiro S, Onodera S and Ikejima T: Reactive oxygen species contribute to oridonin-induced apoptosis and autophagy in human cervical carcinoma HeLa cells. Acta Pharmacol Sin 32: 1266-1275, 2011.

31. Fujii S, Mitsunaga S, Yamazaki M, Hasebe T, Ishii G, Kojima M, Kinoshita T, Ueno T, Esumi $\mathrm{H}$ and Ochiai A: Autophagy is activated in pancreatic cancer cells and correlates with poor patient outcome. Cancer Sci 99: 1813-1819, 2008.

32. Ogata A, Yanagie H, Ishikawa E, Morishita Y, Mitsui S, Yamashita A, Hasumi K, Takamoto S, Yamase T and Eriguchi M: Antitumour effect of polyoxomolybdates: Induction of apoptotic cell death and autophagy in in vitro and in vivo models. Br J Cancer 98: 399-409, 2008.

33. Scherz-Shouval R, Shvets E, Fass E, Shorer H, Gil L and Elazar Z: Reactive oxygen species are essential for autophagy and specifically regulate the activity of Atg4. EMBO J 26: 1749-1760, 2007.

34. Chen Y, Azad MB and Gibson SB: Superoxide is the major reactive oxygen species regulating autophagy. Cell Death Differ 16: 1040-1052, 2009.

35. Bensaad K, Cheung EC and Vousden KH: Modulation of intracellular ROS levels by TIGAR controls autophagy. EMBO J 28: 3015-3026, 2009.

36. Bruno P, Calastretti A, Priulla M, Asnaghi L, Scarlatti F, Nicolin A and Canti G: Cell survival under nutrient stress is dependent on metabolic conditions regulated by Akt and not by autophagic vacuoles. Cell Signal 19: 2118-2126, 2007. 